\title{
An Exploration of Factors Influencing Self-Efficacy in Online Learning: A Systematic Review
}

\author{
https://doi.org/10.3991/ijet.v13i09.8351 \\ Chattavut Peechapol, Jaitip Na-Songkhla ${ }^{(\varpi)}$, Siridej Sujiva, Arthorn Luangsodsai \\ Chulalongkorn University, Bangkok, Thailand \\ jaitipnegmail.com
}

\begin{abstract}
This review examines 12 years of research by focusing on the following question: What are the factors that influence self-efficacy in an online learning environment? There has been a plethora of research concerning self-efficacy. However, few works have focused on the sources of self-efficacy in online-learning environments. Systematic searches of numerous online databases published between 2005 and 2017, which covered factors influencing self-efficacy in online learning context, resulted in the investigation of 25 studies. The data were extracted, organized and analyzed using a narrative synthesis. Results revealed that various factors improved self-efficacy and provided evidence of significant sources of self-efficacy in the context of online learning. Moreover, the investigation provides guidance for further research in designing online learning environments to enhance the self-efficacy of learners.
\end{abstract}

Keywords-Self-efficacy, Online learning, Narrative synthesis, Sources of self-efficacy, Systematic review

\section{Introduction}

Technological advances and easier access to the Internet have led to an increase in online learning compared with traditional learning environments. Online learning offers learning experiences with technology, which provides accessibility, connectivity, flexibility, and ability to promote interactions among learners. As the number of online-learning users continues to increase, there is a need to understand how students can best apply learning strategies to achieve academic success within the online environment.

Self-efficacy is the belief in one's capabilities to organize and execute the requisite actions required to produce particular results [1]. Beliefs about self-efficacy determine level of motivation as reflected in the amount of effort exerted in an endeavor and the length of time devoted to a challenging situation [2]. If persons have a low level of self-efficacy toward a task, they are less likely to exert effort and accomplish the task. Research findings have demonstrated that self-efficacy is a better predictor of academic achievement than other cognitive or affective processes [3]. Therefore, selfefficacy is critical to learning and performance [4]. Student self-efficacy seems particularly important in challenging learning environments, such as an online learn- 
ing one where students lack opportunities to interact with others and as a result can become socially isolated [5], [6]. Also, the drop-out rate among students in online learning environments is higher than that in traditional learning environments [7]. Drop-out rate also related with a lack of self-efficacy [8]. Understanding self-efficacy in online learning is critical to improving online education, which can be a key component of academic success in distance education [4]. However, the focuses of the previous studies were mostly on the situation of self-efficacy in online learning. There have been very few works analyzing factors effecting self-efficacy. As a consequence, the objective of the current review is to examine systematically factors that contribute to self-efficacy in the online learning environment, and which have not previously appeared in open literature.

\section{Systematic review method}

A systematic review was based on PRISMA guidelines [9]. These structures are the guidelines on the systematic review to compare all the data that matches preset criteria to answer specific research questions: What are the factors that influence selfefficacy in an online-learning environment?

\subsection{Search strategy}

An extensive search strategy of the ERIC, Scopus, and Web of Science online databases was conducted and separated into two key search terms. The strategy search terms are shown in Fig. 1.

\begin{tabular}{|c|c|}
\hline \multicolumn{2}{|c|}{$\begin{array}{l}\text { Databases: } \\
\text { Scopus ( } 876 \text { articles), Web of Science ( } 526 \text { articles) and ERIC ( } 772 \text { articles) } \\
\text { Total: } 2174 \text { articles }\end{array}$} \\
\hline Search terms 1 & Search terms 2 \\
\hline $\begin{array}{l}\text { Factor* OR Influence* OR Effect* OR } \\
\text { Affect* OR Role* OR Effect* } \\
\text { AND } \\
\text { "Self-efficacy" } \\
\text { AND } \\
\text { "Online learning" OR "e-learning" OR } \\
\text { "Distance learning" OR "Mobile learn- } \\
\text { ing" OR "Web-based learning" }\end{array}$ & $\begin{array}{l}\text { Correlat* OR Predict* OR Relat* } \\
\text { AND } \\
\text { "Self-efficacy" } \\
\text { AND } \\
\text { "Online learning" OR “e-learning" OR } \\
\text { "Distance learning" OR "Mobile learn- } \\
\text { ing" OR "Web-based learning" }\end{array}$ \\
\hline & \\
\hline
\end{tabular}

Fig. 1. Example of a full-search strategy 


\subsection{Inclusion and Exclusion criteria}

Papers for inclusion in the review were limited to publication in the English language between 2005 and 2017. The final search was conducted in September 2017. The study collected only the research concerned with factors that effect self-efficacy in online learning. One reviewer screened titles and abstracts of studies for first selection. After that, all reviewers examined the remaining full texts of studies to determine eligibility for inclusion in the review. Disagreements between reviewers were resolved through discussion of the degree to which articles met exclusion criteria.

\subsection{Search outcomes}

A total of 25 studies were identified for inclusion in the review. Data from the search strategy of online databases yielded 2174 results. After the removal of duplicates, the remaining 1513 records were assessed based on titles and abstracts. Moreover, 1090 records were excluded from reviewing the titles and abstracts because these studies did not meet inclusion criteria. The full texts of the remaining 69 studies were examined, and 25 were considered relevant. The process used to reduce and evaluate the records is illustrated in the PRISMA flow diagram as displayed in Fig. 2.

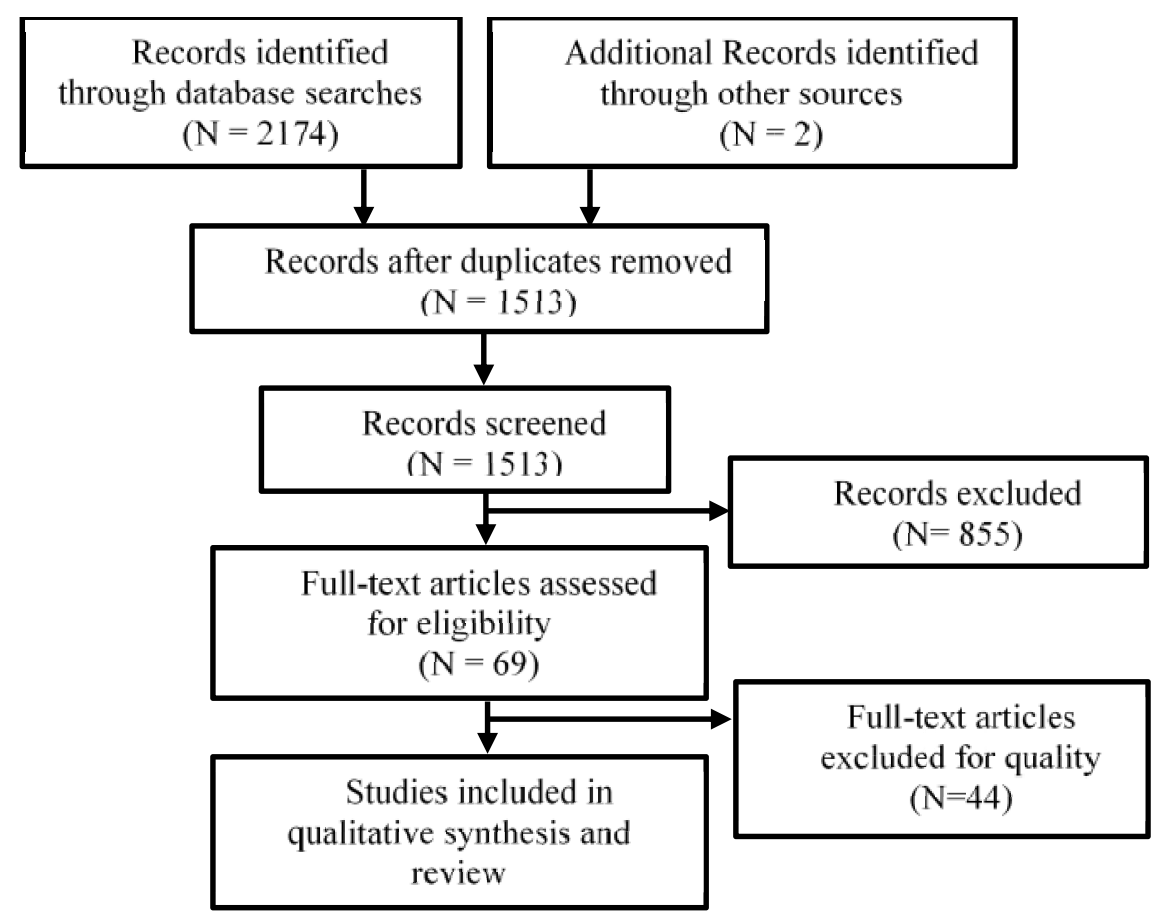

Fig. 2. Flow diagram of studies included in review 


\subsection{Data Extraction and Synthesis}

A data extraction table was developed to enable collection of information relevant to the review. All data were collated and manually synthesized. Information extracted from each included a study of sample characteristics (sample size, mean age, gender, and researched location), study design, factor measures, self-efficacy measures, and relevant findings. In addition, a narrative summary was provided.

\section{Results and Discussion}

The results of the review are presented to explore factors influencing self-efficacy in online learning. Summary of included studies within the systematic review can be seen in Table 1.

\subsection{Study characteristics}

The selected studies encompass research investigated between 2005 and 2010 (eight studies), between 2011 and 2015 (eight studies), and between 2016 and September 2017 (nine studies). The selected-studies research included 22 survey studies and three quasi-experiments.

Table 1. Summary of included studies within the systematic review

\begin{tabular}{|c|c|c|c|}
\hline $\begin{array}{l}\text { Authors } \\
\text { (Year) }\end{array}$ & $\begin{array}{l}\text { Sample Charac- } \\
\text { teristics }\end{array}$ & Study design & Finding \\
\hline $\begin{array}{l}\text { Jashapara and } \\
\text { Tai [10] }\end{array}$ & N: 107 & $\begin{array}{l}\text { Research: Survey } \\
\text { Independent varia- } \\
\text { ble(s): } \\
\text { - Personal innovative- } \\
\text { ness with IT [11] } \\
\text { - Computer playfulness } \\
\text { [12] } \\
\text { - Computer experience } \\
\text { [13], [14], [15] } \\
\text { Mediator variable(s): } \\
\text { - E-learning system self- } \\
\text { efficacy [16] } \\
\text { - Computer anxiety [17] } \\
\text {, [18] } \\
\text { Dependent variable(s): } \\
\text { Perceived ease of use of } \\
\text { e-learning } \\
\text { systems [19], [20] }\end{array}$ & $\begin{array}{l}\text { - Computer experience had a } \\
\text { significant effect on e-learning } \\
\text { specific self-efficacy }(\beta=.51, p \\
<.001) \text {. } \\
\text { - Personal innovativeness with IT } \\
\text { had a significant influence on e- } \\
\text { learning specific self-efficacy ( } \beta \\
=0.46, \mathrm{p}<.001) \text {. } \\
\text { - Computer playfulness had a } \\
\text { significant effect on e-learning } \\
\text { specific self-efficacy ( } \beta=0.40, p \\
<.001) . \\
\text { - E-learning specific self-efficacy } \\
\text { mediated the effect of computer } \\
\text { experience on perceived ease of } \\
\text { use } \\
\text { - E-learning specific self-efficacy } \\
\text { partially mediated the effect of } \\
\text { personal innovativeness and } \\
\text { computer playfulness on per- } \\
\text { ceived ease of use. }\end{array}$ \\
\hline $\begin{array}{l}\text { Bates and } \\
\text { Khasawneh } \\
{[21]}\end{array}$ & $\begin{array}{l}\text { N: } 288 \\
\text { Country: US. } \\
\text { Gender: } \\
\text { - Male: }(28 \%) \\
\text { - Female: }(72 \%)\end{array}$ & $\begin{array}{l}\text { Research: Survey } \\
\text { Independent varia- } \\
\text { ble(s): } \\
\text {-Previous success with } \\
\text { online learning technol- }\end{array}$ & $\begin{array}{l}\text {-Previous success with online } \\
\text { learning technology }(\mathrm{r}=0.48, \mathrm{p} \\
\leq 0.01) \text {, Fixed ability }(\mathrm{r}=-0.32, \\
\mathrm{p} \leq .01) \text {, Acquired skill }(\mathrm{r}=0.38, \\
\mathrm{p} \leq .01) \text {, and online learning }\end{array}$ \\
\hline
\end{tabular}




\begin{tabular}{|c|c|c|c|}
\hline $\begin{array}{l}\text { Authors } \\
\text { (Year) }\end{array}$ & $\begin{array}{l}\text { Sample Charac- } \\
\text { teristics }\end{array}$ & Study design & Finding \\
\hline & $\begin{array}{l}\text { Age: } \\
\text { - Lower-21: (27\%) } \\
-21-29: \\
(57 \%) \\
\text {-29-Upper: }(16 \%)\end{array}$ & $\begin{array}{l}\text { ogy } \\
\text {-Pre-course training } \\
\text {-Instructor feedback } \\
\text { - Fixed ability } \\
\text { - Acquired ability } \\
\text {-Online learning system } \\
\text { anxiety } \\
\text { Mediator variable(s): } \\
\text { Online learning self- } \\
\text { efficacy [22], [23] } \\
\text { Dependent variable(s): } \\
\text { - Outcome expectations } \\
\text { - Skill mastery } \\
\text { - Number of hours spent } \\
\text { per week }\end{array}$ & $\begin{array}{l}\text { system anxiety }(\mathrm{r}=-.56, \mathrm{p} \leq .01) \\
\text { correlated with self-efficacy. } \\
\text { - Previous success had a } \\
\text { significant effect on self-efficacy } \\
(\beta=.2, \mathrm{p} \leq .05) \text {. } \\
\text {-Instructor feedback was consist- } \\
\text { ently significant with self- } \\
\text { efficacy ( } \beta=-.11, \mathrm{p} \leq .05) \text {. } \\
\text {-Acquired skill had a significant } \\
\text { effect on self-efficacy }(\beta=.15, \mathrm{p} \\
\leq .05) \text {. } \\
\text {-Anxiety had a significant effect } \\
\text { on self-efficacy ( } \beta=-.36, \mathrm{p} \leq \\
.05) \text {. } \\
\text {-Online learning self-efficacy } \\
\text { mediates the relationship be- } \\
\text { tween the independent variables } \\
\text { and each of the outcomes. }\end{array}$ \\
\hline $\begin{array}{l}\text { Choi, et al. } \\
{[24]}\end{array}$ & N: 223 & $\begin{array}{l}\text { Research: Survey } \\
\text { Independent varia- } \\
\text { ble(s): } \\
\text { Learner interface, } \\
\text { interaction, instructor } \\
\text { attitude towards stu- } \\
\text { dents, instructor tech- } \\
\text { nical competence and } \\
\text { content [25], [26] } \\
\text { Mediator variable(s): } \\
\text { - Attitude towards e- } \\
\text { learning [27] } \\
\text { - Flow experience [28] } \\
\text { Dependent variable(s): } \\
\text { Technology self- } \\
\text { efficacy in ERP system } \\
\text { usage [29] }\end{array}$ & $\begin{array}{l}\text { - The effect of flow experience } \\
\text { on technology self-efficacy in } \\
\text { ERP system usage was supported } \\
\text { at } 99 \% \text { (path coefficient }=0.296 \text {, } \\
\mathrm{t}=4.123 \text { ). } \\
\text { - Attitude towards } \\
\text { learning had a significant effect } \\
\text { on technology self-efficacy in } \\
\text { ERP system (path coefficient }= \\
0.323, \mathrm{t}=3.864 \text { ). }\end{array}$ \\
\hline $\begin{array}{l}\text { Wang and } \mathrm{Wu} \\
{[30]}\end{array}$ & $\begin{array}{l}\text { N: } 76 \\
\text { Country: } \\
\text { Taiwan }\end{array}$ & $\begin{array}{l}\text { Design: quasi- } \\
\text { experiment } \\
\text { Independent varia- } \\
\text { ble(s): } \\
\text { - Receiving elaborate } \\
\text { feedback } \\
\text { Dependent variable(s): } \\
\text { - Self-efficacy [31] } \\
\text { - Academic perfor- } \\
\text { mance }\end{array}$ & $\begin{array}{l}\text { Receiving elaborate feedback } \\
\text { was significantly related to the } \\
\text { difference between students' } \\
\text { self-efficacy on original assign- } \\
\text { ment and self-efficacy on revised } \\
\text { assignment }(\beta=.287, \mathrm{p}<.05) \text {. }\end{array}$ \\
\hline Chu [32] & $\begin{array}{l}\text { N: } 290 \\
\text { Country: } \\
\text { Taiwan } \\
\text { Gender: } \\
\text { - Male: } 112(39 \%) \\
\text { - Female: } 178(61 \%) \\
\text { Age: } \\
\text { - 50-64: } 215 \\
(74.14 \%)\end{array}$ & $\begin{array}{l}\text { Research: Survey } \\
\text { Independent varia- } \\
\text { ble(s): } \\
\text { - Tangible family } \\
\text { support } \\
\text { - Emotional family } \\
\text { support } \\
\text { Mediator variable(s): } \\
\text { Internet self-efficacy } \\
\end{array}$ & $\begin{array}{l}\text { - Age }(\mathrm{r}=.20, \mathrm{p}<.05), \text { tangible } \\
\text { family support } \\
(\mathrm{r}=.30, \mathrm{p}<.01), \text { and emotional } \\
\text { family support }(\mathrm{r}=.39, \mathrm{p}<.01) \\
\text { significantly correlated with } \\
\text { general internet self-efficacy. } \\
\text { - Tangible }(\mathrm{r}=.22, \mathrm{p}<.01) \text { and } \\
\text { emotional family support }(\mathrm{r}=.36 \text {, } \\
\mathrm{p}<.05)\end{array}$ \\
\hline
\end{tabular}




\begin{tabular}{|c|c|c|c|}
\hline $\begin{array}{l}\text { Authors } \\
\text { (Year) }\end{array}$ & $\begin{array}{l}\text { Sample Charac- } \\
\text { teristics }\end{array}$ & Study design & Finding \\
\hline & $\begin{array}{l}\text { - 64-Upper: } 75 \\
(25.86 \%) \\
\text { Mean: } 58.59, \mathrm{SD}= \\
5.78\end{array}$ & $\begin{array}{l}\text { [33] } \\
\text { Dependent variable(s): } \\
\text { The effects of e-learning } \\
\text { (Perceived learning, } \\
\text { Intent-to-persist in e- } \\
\text { learning, and learning } \\
\text { satisfaction [34] }\end{array}$ & $\begin{array}{l}\text { significantly correlated with } \\
\text { communication internet self- } \\
\text { efficacy. } \\
\text { - Emotional family support } \\
\text { contributed significantly to the } \\
\text { prediction of general internet } \\
\text { self-efficacy }(\beta=.38, \mathrm{p}<.01) \\
\text { and communication internet self- } \\
\text { efficacy }(\beta=.20, \mathrm{p}<.01) \text {. } \\
\\
\text { - Tangible family support con- } \\
\text { tributed significantly to the } \\
\text { prediction of general internet } \\
\text { self-efficacy }(\beta=.17, \mathrm{p}<.01) \\
\text { and communication internet self- } \\
\text { efficacy }(\beta=.16, p<.01) \text {. }\end{array}$ \\
\hline $\begin{array}{l}\text { Chu and Chu } \\
\text { [35] }\end{array}$ & $\begin{array}{l}\text { N: } 317 \\
\text { Country: } \\
\text { Taiwan } \\
\text { Gender: } \\
\text { - Male: } 111(35.04 \%) \\
\text { - Female: } 206 \\
(64.96 \%) \\
\text { Age: } \\
\text { Mean: } 54.59\end{array}$ & $\begin{array}{l}\text { Research: Survey } \\
\text { Independent varia- } \\
\text { ble(s): } \\
\text { - Peer support [36] } \\
\text { Mediator variable(s): } \\
\text { Internet self-efficacy } \\
\text { [33] } \\
\text { Moderator(s): } \\
\text { - Aggregate collectiv- } \\
\text { ism [37] } \\
\text { - Aggregate group } \\
\text { potency } \\
\text { [37], [38] } \\
\text { Dependent variable(s): } \\
\text { The e-learning outcome: } \\
\text { perceived learning, } \\
\text { persistence and satisfac- } \\
\text { tion [34] }\end{array}$ & $\begin{array}{l}\text { - Age }(\mathrm{r}=-.32, \mathrm{p}<.01), \text { Peer } \\
\text { support }(\mathrm{r}=-.38, \mathrm{p}<.01), \text { Col- } \\
\text { lectivism }(\mathrm{r}=0.20, \mathrm{p}<.01), \text { and } \\
\text { Group potency }(\mathrm{r}=.22, \mathrm{p}<.01) \\
\text { were significantly associated } \\
\text { with internet self-efficacy. } \\
\text { - Collectivism }(\mathrm{r}=0.20, \mathrm{p}<.01) \\
\text { and } \\
\text { Group potency }(\mathrm{r}=.22, \mathrm{p}<.01) \\
\text { were significantly associated } \\
\text { with internet self-efficacy. } \\
\text { - The effect of peer support on } \\
\text { internet self-efficacy was signifi- } \\
\text { cant }(\gamma=.36, \mathrm{p}<.01) \text {. } \\
\text { - Collectivism significantly } \\
\text { moderated the cross-level inter- } \\
\text { action between peer support and } \\
\text { internet self-efficacy }(\gamma=.27, \mathrm{p}< \\
.01) \text {. }\end{array}$ \\
\hline $\begin{array}{l}\text { Law, Lee and } \\
\text { Yu [39] }\end{array}$ & $\begin{array}{l}\text { N: } 365 \\
\text { Country: Hong } \\
\text { Kong } \\
\text { Gender: } \\
\text { - Male: } 254(69.6 \%) \\
\text { - Female: } 111 \\
(30.4 \%)\end{array}$ & $\begin{array}{l}\text { Design: Survey } \\
\text { Independent varia- } \\
\text { ble(s): } \\
\text { - Individual attitude and } \\
\text { Expectation } \\
\text { - Reward and recogni- } \\
\text { tion } \\
\text { - Punishment } \\
\text { - Challenging goals } \\
\text { - Social pressure and } \\
\text { competition } \\
\text { Dependent variable(s): } \\
\text { Self-efficacy }\end{array}$ & $\begin{array}{l}\text { - Individual attitude and expecta- } \\
\text { tion }(\mathrm{r}=.57, \mathrm{p}<.01), \text { Challeng- } \\
\text { ing goals }(\mathrm{r}=.66, \mathrm{p}<.01), \text { Clear } \\
\text { direction }(\mathrm{r}=.52, \mathrm{p}<.01), \\
\text { Reward and recognition }(\mathrm{r}=.52 \text {, } \\
\mathrm{p}<.01), \text { Punishment }(\mathrm{r}=.42, \mathrm{p}< \\
.01), \text { and Social pressure and } \\
\text { competition }(\mathrm{r}=.57, \mathrm{p}<.01) \\
\text { correlated with self-efficacy. } \\
\text { - Individual attitude and expecta- } \\
\text { tion }(\beta=0.122, \mathrm{p}<.01), \text { chal- } \\
\text { lenging goals }(\beta=0.429, \mathrm{p}< \\
.01), \text { and social pressure and } \\
\text { competition }(\beta=0.262, \mathrm{p}<.01) \\
\text { had a significant effect on self- } \\
\text { efficacy. }\end{array}$ \\
\hline $\begin{array}{l}\text { Tseng and } \\
\text { Kuo [40] }\end{array}$ & $\begin{array}{l}\text { N: - } \\
\text { Country: } \\
\text { Taiwan }\end{array}$ & $\begin{array}{l}\text { Design: Survey } \\
\text { Independent varia- } \\
\text { ble(s): }\end{array}$ & $\begin{array}{l}\text { - Community identity had a } \\
\text { significant positive effect on } \\
\text { knowledge-sharing self-efficacy }\end{array}$ \\
\hline
\end{tabular}




\begin{tabular}{|c|c|c|c|}
\hline $\begin{array}{l}\text { Authors } \\
\text { (Year) }\end{array}$ & $\begin{array}{l}\text { Sample Charac- } \\
\text { teristics }\end{array}$ & Study design & Finding \\
\hline & & $\begin{array}{l}\text { - Community identity } \\
\text { [41], [42] } \\
\text { - Interpersonal trust [43] } \\
\text {, [44], [45] } \\
\text { Mediator variable(s): } \\
\text { - Social awareness [46], } \\
\text { [47] } \\
\text { - Knowledge-sharing } \\
\text { self-efficacy [48], [49], } \\
\text { [50], [51] } \\
\text { Dependent variable(s): } \\
\text { Knowledge-sharing } \\
\text { behavior [52], [53] }\end{array}$ & $\begin{array}{l}(\beta=0.37, \mathrm{p}<0.05) \\
\text {-Effect of interpersonal trust }(\beta= \\
0.30, \mathrm{p}<0.01) \text { on knowledge- } \\
\text { sharing self-efficacy was signifi- } \\
\text { cant. } \\
\text { - Community identity and inter- } \\
\text { personal trust influenced } \\
\text { knowledge-sharing behavior } \\
\text { through the mediation of } \\
\text { knowledge sharing self-efficacy. }\end{array}$ \\
\hline $\begin{array}{l}\text { Jashapara and } \\
\text { Tai [54] }\end{array}$ & $\begin{array}{l}\text { N: } 403 \\
\text { Country: } \\
\text { Gender: } \\
\text { - Male: } \\
204(50.6 \%) \\
\text { - Female: } \\
\text { 199 }(49.4 \%) \\
\text { Age: } \\
\text { Mean: } 23\end{array}$ & $\begin{array}{l}\text { Design: Survey } \\
\text { Factor(s): } \\
\text { - Personal innovative- } \\
\text { ness with IT [11] } \\
\text { - Computer playfulness } \\
\text { [12] } \\
\text { Computer experience } \\
\text { [13], [14], [15] } \\
\text { Mediator: } \\
\text { - Computer Anxiety } \\
\text { [18], [55] } \\
\text { - E-learning system self- } \\
\text { efficacy [16] } \\
\\
\text { Dependent variable(s): } \\
\text { Perceived Ease of Use } \\
\text { [20] }\end{array}$ & $\begin{array}{l}\text { - Personal innovativeness with IT } \\
\text { showed significant effect on e- } \\
\text { learning system self-efficacy ( } \beta= \\
0.34, p<0.001) \text {. } \\
\text { - Computer playfulness ( } \beta= \\
0.18, p<0.001) \text { had a significant } \\
\text { positive effect on e-learning } \\
\text { system self-efficacy. } \\
\text { - Computer experience ( } \beta=0.39 \text {, } \\
\text { p }<0.001) \text { had a significant } \\
\text { effect on e-learning system self- } \\
\text { efficacy. } \\
\text { - E-learning system self-efficacy } \\
\text { completely mediated the effects } \\
\text { of } \\
\text { computer experience and com- } \\
\text { puter playfulness on perceived } \\
\text { ease of use } \\
\text { - E-learning system self-efficacy } \\
\text { partially mediated the effect of } \\
\text { personal innovativeness with IT } \\
\text { on perceived ease of use. }\end{array}$ \\
\hline $\begin{array}{l}\text { Zang, et al. } \\
{[56]}\end{array}$ & $\begin{array}{l}\text { N: } 144 \\
\text { Country: Hong } \\
\text { Kong } \\
\text { Gender: } \\
\text { - Male: } 114(79.2 \%) \\
\text { - Female: } 30(20.8 \%) \\
\text { Age: } \\
\text { - } 25 \text {-: } 17(11.8 \%) \\
\text { - 25-32: } 94(65.3 \%) \\
\text { - 33-40: } 25(17.3 \%) \\
\text { - 40-Upper: } 8(5.6 \%)\end{array}$ & $\begin{array}{l}\text { Design: Survey } \\
\text { Independent varia- } \\
\text { ble(s): } \\
\text { Environmental fac- } \\
\text { tors: } \\
\text { - Perceived responsive- } \\
\text { ness [44] } \\
\text { - Psychological safety } \\
\text { communication climate } \\
\text { [57] } \\
\text { Person factor: } \\
\text { - Self-efficacy [58] } \\
\text { Dependent variable(s): } \\
\text { - Satisfaction [59] } \\
\text { - Intention to continue } \\
\text { participation [59], [60] }\end{array}$ & $\begin{array}{l}\text { - Perceived responsiveness was } \\
\text { observed to influence self- } \\
\text { efficacy significantly (path } \\
\text { coefficient }=0.20, \mathrm{p}<0.01 \text { ) } \\
\text { - The results revealed the posi- } \\
\text { tive influence of the psychologi- } \\
\text { cal-safety communication cli- } \\
\text { mate on self-efficacy (path } \\
\text { coefficient }=0.30, \mathrm{p}<0.01 \text { ) } \\
\text { - Self-efficacy partially mediated } \\
\text { the relationship between the } \\
\text { psychological-safety communi- } \\
\text { cation climate and the intention } \\
\text { to continue participation (Sobel } \\
\text { statistics }=2.07, \mathrm{p}=.038 \text { ). }\end{array}$ \\
\hline $\begin{array}{l}\text { Shen, et al. } \\
\text { [61] }\end{array}$ & $\begin{array}{l}\text { N: } 406 \\
\text { Country: U.S. } \\
\text { Gender: } \\
\text { - Male: } 104(25.16 \%)\end{array}$ & $\begin{array}{l}\text { Design: Survey } \\
\text { Independent varia- } \\
\text { ble(s): } \\
\text { Number of Online }\end{array}$ & $\begin{array}{l}\text { The number of online courses } \\
\text { was a significant predictor of } \\
\text { self-efficacy to complete an } \\
\text { online course }(\mathrm{t}=3.48, \mathrm{p}<.01)\end{array}$ \\
\hline
\end{tabular}




\begin{tabular}{|c|c|c|c|}
\hline $\begin{array}{l}\text { Authors } \\
\text { (Year) }\end{array}$ & $\begin{array}{l}\text { Sample Charac- } \\
\text { teristics }\end{array}$ & Study design & Finding \\
\hline & $\begin{array}{l}\text { - Female: } 301 \\
(74.14 \%) \\
\text { - No response: } 1 \\
(0.7 \%)\end{array}$ & $\begin{array}{l}\text { Courses } \\
\text { Dependent variable(s): } \\
\text { - Dimensions of online } \\
\text { learning self-efficacy } \\
\text { - Online learning satis- } \\
\text { faction }\end{array}$ & \\
\hline $\begin{array}{l}\text { Wang, et al. } \\
{[62]}\end{array}$ & $\begin{array}{l}\text { N: } 256 \\
\text { Country: US. } \\
\text { Gender: } \\
\text { - Male: } 121(47.3 \%) \\
\text { - Female: } 135 \\
(53.1 \%)\end{array}$ & $\begin{array}{l}\text { Design: Survey } \\
\text { Independent varia- } \\
\text { ble(s): } \\
\text { - Gender } \\
\text { - Education level } \\
\text { - Previous experience } \\
\text { Mediator variable(s): } \\
\text { - Motivation and learn- } \\
\text { ing strategies (Modified } \\
\text { motivation strategies for } \\
\text { learning questionnaire } \\
\text { [63] } \\
\text { - Online technology } \\
\text { self-efficacy [64] } \\
\text { Dependent variable(s): } \\
\text { - Achievement } \\
\text { - Students' overall } \\
\text { satisfaction with the } \\
\text { online courses [65] }\end{array}$ & $\begin{array}{l}\text { - Motivation directly influenced } \\
\text { the levels of technology self- } \\
\text { efficacy }(\beta<.796, p<.001) \text {. } \\
\text { - Motivation was the mediator } \\
\text { between } \\
\text { the learning strategies and tech- } \\
\text { nology self-efficacy }\end{array}$ \\
\hline $\begin{array}{l}\text { Chiu and Tsai } \\
{[66]}\end{array}$ & $\begin{array}{l}\text { N: } 244 \\
\text { (All female) } \\
\text { Country:Taiwan } \\
\text { Age: } \\
\text { - 21-30: } 119(48.8 \%) \\
\text { - 31-40: } 76(31.1 \%) \\
\text { - 41-50: } 40(16.4 \%)\end{array}$ & $\begin{array}{l}\text { Design: Survey } \\
\text { Independent varia- } \\
\text { ble(s): } \\
\text { - Social Factor: manage- } \\
\text { rial support, job support } \\
\text { and organizational } \\
\text { support [67] } \\
\text { - Personal factor: Inter- } \\
\text { net Self-efficacy (Liang, } \\
\text { Wu and Tsai, 2011) } \\
\text { Dependent variable(s): } \\
\text { Attitudes towards web- } \\
\text { based continued learn- } \\
\text { ing [68] }\end{array}$ & $\begin{array}{l}\text { - Social factor correlated with } \\
\text { basic internet self-efficacy }(\mathrm{r}= \\
.29, \mathrm{p}<.001) \text { and advanced } \\
\text { internet self-efficacy }(\mathrm{r}=.37, \mathrm{p}< \\
.001) \\
\text { - The social factor had positive } \\
\text { effects on basic internet self- } \\
\text { efficacy }(\gamma=.37, \mathrm{p}<.001) \text { and } \\
\text { advanced internet self-efficacy }(\gamma \\
=.29, \mathrm{p}<.001) \\
\text { - Social factor played an indirect } \\
\text { role in nurses' intention to use } \\
\text { web-based continued learning } \\
\text { through basic internet self- } \\
\text { efficacy. }\end{array}$ \\
\hline $\begin{array}{l}\text { Tang, et al. } \\
\text { [69] }\end{array}$ & $\begin{array}{l}\text { N: } 318 \\
\text { Country: } \\
\text { Chinese } \\
\text { Gender: } \\
\text { - Male: } 130(40.9 \%) \\
\text { - Female: } 188 \\
\text { (59.1\%) } \\
\text { Age: } \\
\text { - 23: } 151(47.5 \%) \\
\text { - 23-30: } 145(45.6 \%) \\
\text { - 31-40: } 15(4.70 \%) \\
\text { - 41-50: } 6(1.90 \%) \\
\text { - 50-Upper: } 1 \\
(0.30 \%)\end{array}$ & $\begin{array}{l}\text { Design: Survey } \\
\text { Independent varia- } \\
\text { ble(s): } \\
\text { - The expectation- } \\
\text { confirmation model } \\
{[70],[71]} \\
\text { - Experiential [72], [73] } \\
\text { - Perceived usefulness } \\
\text { [74] } \\
\text { - Perceived self-efficacy } \\
\text { [1], [75] } \\
\text { Dependent variable(s): } \\
\text { Intention to continue } \\
\text { learning [70] }\end{array}$ & $\begin{array}{l}\text { - Confirmation was significantly } \\
\text { related to perceived self-efficacy } \\
(\beta=0.819, t=15.588)\end{array}$ \\
\hline
\end{tabular}




\begin{tabular}{|c|c|c|c|}
\hline $\begin{array}{l}\text { Authors } \\
\text { (Year) }\end{array}$ & $\begin{array}{l}\text { Sample Charac- } \\
\text { teristics }\end{array}$ & Study design & Finding \\
\hline Lin, et al. [76] & $\begin{array}{l}\text { N: } 210 \\
\text { Gender: } \\
\text { - Male: } 122(58.10 \%) \\
\text { - Female: } 88(41.9 \%) \\
\text { Age: } \\
\text { - 21-30: } 121(57.6 \%) \\
\text { - 30-40: } 89(30.5 \%)\end{array}$ & $\begin{array}{l}\text { Design: Survey } \\
\text { Independent varia- } \\
\text { ble(s): } \\
\text { - Teaching presence - } \\
\text { Social presence } \\
\text { Mediator variable(s): } \\
\text { - Self-efficacy } \\
\text { - Cognitive Presence } \\
\text { Dependent variable(s): } \\
\text { Training Effectiveness }\end{array}$ & $\begin{array}{l}\text { - Teaching presence significantly } \\
\text { predicted self-efficacy }(\beta= \\
.0217, \mathrm{t}=2.503, \mathrm{p}<.05) \\
\text { - Social presence was a stronger } \\
\text { predictor of self-efficacy }(\beta= \\
.477, \mathrm{t}=5.077, \mathrm{p}<.001) . \\
\text { - Self-efficacy is a full mediator } \\
\text { between social presence and } \\
\text { cognitive presence. }\end{array}$ \\
\hline Shen [77] & $\mathbf{N}: 250$ & $\begin{array}{l}\text { Design: Survey } \\
\text { Independent varia- } \\
\text { ble(s): } \\
\text { - Sense of community } \\
\text { [78] } \\
\text { - Community trust [79] } \\
\text { - Self-efficacy [58] } \\
\text { Dependent variable(s): } \\
\text { Knowledge Sharing }\end{array}$ & $\begin{array}{l}\text { - Trust between member had a } \\
\text { significant positive effect on self- } \\
\text { efficacy of knowledge-sharing ( } \\
\beta=0.405, p<.001) \\
\text {-Effect of perceptual learning ( } \beta \\
=0.433, p<.001 \text { ) on self- } \\
\text { efficacy of knowledge-sharing } \\
\text { was significant. }\end{array}$ \\
\hline $\begin{array}{l}\text { Lim, Kang } \\
\text { and Park [80] }\end{array}$ & $\begin{array}{l}\text { N: } 937 \\
\text { Country: Korea } \\
\text { Gender: } \\
\text { - Male: } 407(43.44 \%) \\
\text { - Female: } 503 \\
(56.56 \%) \\
\text { Age: } \\
\text { - 20: } 10 \\
(1.07 \%) \\
\text { - 20-29: } 189 \\
(20.17 \%) \\
-30-39: 264(28.17) \\
-40-49: 301 \\
(32.12 \%) \\
-49-U p p e r: 173 \\
(18.46 \%)\end{array}$ & $\begin{array}{l}\text { Design: Survey } \\
\text { Independent varia- } \\
\text { ble(s): } \\
\text { - Learner-learner inter- } \\
\text { action and } \\
\text { learner-instructor inter- } \\
\text { action [81] } \\
\text { - The quality of learning } \\
\text { content [82], [83] } \\
\text { - The quality of online } \\
\text { learning systems used } \\
\text { by participants [84] } \\
\text { - Extrinsic and Intrinsic } \\
\text { motivation [85] } \\
\text { - Computer self-efficacy } \\
\text { [86] } \\
\text { - Academic self- } \\
\text { efficacy [87] } \\
\text { Dependent variable(s): } \\
\text { - Class satisfaction } \\
\text { - Academic achieve- } \\
\text { ment }\end{array}$ & $\begin{array}{l}\text { - Learner-learner interaction }(\mathrm{r}= \\
0.28) \text { and system quality }(\mathrm{r}= \\
0.18) \text { was related to learner } \\
\text { computer self-efficacy } \\
\text { - Learner-learner interaction }(\mathrm{r}= \\
0.59) \text { and content quality }(\mathrm{r}= \\
0.45) \text { was related to learner } \\
\text { academic self-efficacy } \\
\text { - Learner-learner interaction had } \\
\text { a significant effect on both } \\
\text { computer self-efficacy }(\beta=.28, \mathrm{p} \\
<.001) \text { and academic self- } \\
\text { efficacy }(\beta=.56, \mathrm{p}<.001) \\
\text { - Content quality significantly } \\
\text { predicted computer self-efficacy } \\
(\beta=.23, \mathrm{p}<.001) \text {. } \\
\text { - System quality significantly } \\
\text { effected academic self-efficacy } \\
(\beta=.37, \mathrm{p}<.001) \text {. }\end{array}$ \\
\hline $\begin{array}{l}\text { Liou, et al. } \\
{[88]}\end{array}$ & $\begin{array}{l}\text { N: } 394 \\
\text { Country: } \\
\text { Taiwan } \\
\text { Gender: } \\
\text { - Male: } 128(32.48 \%) \\
\text { - Female: } 266 \\
(67.52 \%) \\
\text { Age: } \\
\text { - } 21-24: 121 \\
(30.66 \%) \\
-25-34: 273 \\
(55.47 \%)\end{array}$ & $\begin{array}{l}\text { Design: Survey } \\
\text { Independent varia- } \\
\text { ble(s): } \\
\text { - The anticipated recip- } \\
\text { rocal relationship and } \\
\text { anticipated extrinsic } \\
\text { rewards [89] } \\
\text { - Norm of reciprocity } \\
\text { [58] } \\
\text { Mediator variable(s): } \\
\text { - Knowledge sharing } \\
\text { self-efficacy [58] } \\
\text { - Three items for } \\
\text { knowledge sharing } \\
\text { behavior [90] }\end{array}$ & $\begin{array}{l}\text { - Anticipated reciprocal relation- } \\
\text { ship }(\mathrm{r}=.506, \mathrm{p}<.001), \text { Norm } \\
\text { of reciprocity }(\mathrm{r}=.384, \mathrm{p}< \\
.001) \text {, and Anticipated extrinsic } \\
\text { rewards }(\mathrm{r}=.456, \mathrm{p}<.001) \text { were } \\
\text { significantly correlated with } \\
\text { knowledge sharing self-efficacy } \\
\text { - Anticipated extrinsic rewards } \\
\text { had a significant and positive } \\
\text { effect on knowledge sharing self- } \\
\text { efficacy }(\gamma=0.589, \mathrm{p}<0.001) \\
\text { - The knowledge sharing self- } \\
\text { efficacy partially mediated } \\
\text { knowledge sharing behavior. }\end{array}$ \\
\hline
\end{tabular}




\begin{tabular}{|c|c|c|c|}
\hline $\begin{array}{l}\text { Authors } \\
\text { (Year) }\end{array}$ & $\begin{array}{l}\text { Sample Charac- } \\
\text { teristics }\end{array}$ & Study design & Finding \\
\hline & & $\begin{array}{l}\text { Dependent variable(s): } \\
\text { - Community participa- } \\
\text { tion [91] }\end{array}$ & \\
\hline $\begin{array}{l}\text { Prior, et al. } \\
{[92]}\end{array}$ & $\begin{array}{l}\text { N: } 150 \\
\text { Gender: } \\
\text { - Male: } 102(68 \%) \\
\text { - Female: } 48(32 \%) \\
\text { Age: } \\
\text { - 21-30: } 33(22.4 \%) \\
\text { - 31-40: } 60(39.7 \%) \\
\text { - 41-50: } 40(26.5 \%) \\
\text { - 51-60: } 14(9.3 \%)\end{array}$ & $\begin{array}{l}\text { Design: Survey } \\
\text { Independent varia- } \\
\text { ble(s): } \\
\text { Attitude and Digital } \\
\text { literacy [93] } \\
\text { Mediator variable(s): } \\
\text { Self-efficacy [61] } \\
\text { Dependent variable(s): } \\
\text { - Peer engagement } \\
\text { - Learning-management } \\
\text { system interactions } \\
\text { - Convener interaction }\end{array}$ & $\begin{array}{l}\text { - Attitude }(\mathrm{r}=.577, \mathrm{p}<.01) \text { and } \\
\text { Digital literacy }(\mathrm{r}=.538, \mathrm{p}<.01) \\
\text { were significantly correlated } \\
\text { with self-efficacy. } \\
\text { - Attitude had a significant } \\
\text { positive effect on self-efficacy }(\beta \\
=0.556, \mathrm{p}<0.01) \\
\text { - Digital literacy appears to have } \\
\text { had a significant positive effect } \\
\text { on self-efficacy }(\beta=0.274, \mathrm{p}< \\
0.05) \\
\text { - Self-efficacy had a significant } \\
\text { positive effect on peer engage- } \\
\text { ment }(\beta=.694, \mathrm{p}<.01), \text { Learn- } \\
\text { ing-management system interac- } \\
\text { tions }(\beta=.570, \mathrm{p}<.01) \text { and } \\
\text { convener interaction }(\beta=.646, \mathrm{p} \\
<.01) .\end{array}$ \\
\hline $\begin{array}{l}\text { Reychav, et } \\
\text { al. [94] }\end{array}$ & $\begin{array}{l}\text { N: } 1111 \\
\text { Gender: } \\
\text { - Male: }(52 \%) \\
\text { - Female: }(48 \%) \\
\text { Mean age: } 13.21\end{array}$ & $\begin{array}{l}\text { Design: quasi- } \\
\text { experiment Independ- } \\
\text { ent variable(s): } \\
\text { - Network reciprocity } \\
\text { - Eigenvector centrality } \\
\text { Mediator variable(s): } \\
\text { - Perceived ease of use } \\
\text { and attitude toward } \\
\text { technology [74] } \\
\text { - Computer self-efficacy } \\
\text { [95] } \\
\text { - Perceived enjoyment } \\
\text { [96] } \\
\text { Dependent variable(s): } \\
\text { - Attitude toward Tech- } \\
\text { nology Use } \\
\text { - Performance }\end{array}$ & $\begin{array}{l}\text { - Network reciprocity had a } \\
\text { strong and significant effect on } \\
\text { computer self-efficacy }(\beta=.16, p \\
<.01) \\
\text { - Perceived enjoyment had a } \\
\text { positive effect on computer self- } \\
\text { efficacy }(\beta=.34, p<.01) \text {. }\end{array}$ \\
\hline $\begin{array}{l}\text { Song, et al. } \\
{[97]}\end{array}$ & $\begin{array}{l}\text { N: } 386 \\
\text { Gender: } \\
\text { - Male: } 201(52.1 \%) \\
\text { - Female: } 185 \\
(47.9 \%) \\
\text { Country: US. } \\
\text { Age: } \\
\text { Mean }=25\end{array}$ & $\begin{array}{l}\text { Design: quasi- } \\
\text { experiment } \\
\text { Independent varia- } \\
\text { ble(s): } \\
\text { - Prior knowledge } \\
\text { - Self-regulation } \\
\text { [97] } \\
\text { Dependent variable(s): } \\
\text { - Goal orientation [98] } \\
\text {-Task value [99], [100] } \\
\text { - Self-efficacy [101] } \\
\text { - Clinical reasoning } \\
\text { [102] } \\
\text { Dependent variable(s): } \\
\text { Learning performance }\end{array}$ & $\begin{array}{l}\text { Prior knowledge showed a } \\
\text { positive direct effect on self- } \\
\text { efficacy }(\beta=.3, p<.001)\end{array}$ \\
\hline Vayre and & $\mathbf{N}: 255$ & Design: Survey & - The sense of belonging to a \\
\hline
\end{tabular}




\begin{tabular}{|c|c|c|c|}
\hline $\begin{array}{l}\text { Authors } \\
\text { (Year) }\end{array}$ & $\begin{array}{l}\text { Sample Charac- } \\
\text { teristics }\end{array}$ & Study design & Finding \\
\hline $\begin{array}{l}\text { Vonthron } \\
\text { [103] }\end{array}$ & $\begin{array}{l}\text { Gender: } \\
\text { - Male: } 63(24.7 \%) \\
\text { - Female: } 192 \\
(75.3 \%) \\
\text { Age: } \\
18-68(\text { Mean }= \\
31.60, \mathrm{SD}=10.70)\end{array}$ & $\begin{array}{l}\text { Independent varia- } \\
\text { ble(s): } \\
\text { - Perceived social } \\
\text { support. } \\
\text { - Sense of belonging to } \\
\text { a community [104], } \\
\text { [105] } \\
\text { Mediator variable(s): } \\
\text { Academic Self-efficacy } \\
\text { [106] } \\
\text { Dependent variable(s): } \\
\text { Academic engagement } \\
\text { [107] }\end{array}$ & $\begin{array}{l}\text { community played a significant } \\
\text { and positive role on self-efficacy. } \\
(\beta=0.37, p<0.01) \\
\text { - Academic self-efficacy partial- } \\
\text { ly mediated between the sense of } \\
\text { belonging to the learning com- } \\
\text { munity and enthusiasm }\end{array}$ \\
\hline $\begin{array}{l}\text { Cho and Cho } \\
{[108]}\end{array}$ & $\begin{array}{l}\text { N: } 799 \\
\text { Gender: } \\
\text { - Male: } 247(30.9 \%) \\
\text { - Female: } 552 \\
(69.1 \%)\end{array}$ & $\begin{array}{l}\text { Design: Survey } \\
\text { Independent varia- } \\
\text { ble(s): } \\
\text { SR in the three types of } \\
\text { online interaction (The } \\
\text { online } \\
\text { self-regulation ques- } \\
\text { tionnaire [OSRQ]) } \\
\text { Dependent variable(s): } \\
\text { - Self-efficacy for } \\
\text { learning [109] } \\
\text { - Course satisfaction } \\
\text { scale [110] }\end{array}$ & $\begin{array}{l}\text { - Self-regulation in interaction } \\
\text { between student and content, } \\
\text { student and student and, student } \\
\text { and teacher significantly corre- } \\
\text { lated with self-efficacy }(\mathrm{r}=.52, \mathrm{p} \\
<.001 ; \mathrm{r}=.27, \\
\mathrm{p}<.001 ; \mathrm{r}=.51, \mathrm{p}<.001), \\
\text { respectively. } \\
\text { - Self-regulation in interaction } \\
\text { between student and content ( } \beta= \\
.37, \mathrm{p}<.001) \text { had a positive } \\
\text { effect on self-efficacy for learn- } \\
\text { ing. } \\
\text { - Self-regulation in interaction } \\
\text { between student and teacher in } \\
\text { online courses }(\beta=.30, \mathrm{p}<.001) \\
\text { positively affected self-efficacy } \\
\text { for learning. }\end{array}$ \\
\hline $\begin{array}{l}\text { Hong, et al. } \\
\text { [111] }\end{array}$ & $\begin{array}{l}\text { N: } 73 \\
\text { Country: } \\
\text { Taiwan } \\
\text { Gender: } \\
\text { - Male: } 34(46.6 \%) \\
\text { - Female: } 39(53.4 \%) \\
\text { Age: } \\
\text { Mean: } 10.62\end{array}$ & $\begin{array}{l}\text { Design: quasi- } \\
\text { experiment } \\
\text { Independent varia- } \\
\text { ble(s): } \\
\text { - Intrinsic motivation of } \\
\text { Chinese learning } \\
\text { Mediator variable(s): } \\
\text { - Online learning self- } \\
\text { efficacy } \\
\text { - Flow experience } \\
\text { Dependent variable(s): } \\
\text { Degree of learning } \\
\text { progress }\end{array}$ & $\begin{array}{l}\text { - The relationship between } \\
\text { Chinese learning intrinsic moti- } \\
\text { vation and online learning self- } \\
\text { efficacy was supported with a } \\
\text { path coefficient of } .382(\mathrm{t}=4.35 \text {, } \\
\mathrm{p}<0.001) \\
\text { - The test of the relationship } \\
\text { between the degree of progress } \\
\text { and online learning self-efficacy } \\
\text { was supported by a path coeffi- } \\
\text { cient of } .222(\mathrm{t}=2.37, \mathrm{p}<.05)\end{array}$ \\
\hline $\begin{array}{l}\text { Kim and Park } \\
{[112]}\end{array}$ & $\begin{array}{l}\text { N: } 707 \\
\text { (Learner: } 384 \\
\text {, Instructor: } 353) \\
\text { Gender: } \\
\text { Learner } \\
\text { - Male: } 219(62.04 \%) \\
\text { - Female: } 134 \\
\text { (37.96\%) } \\
\text { Instructor: } \\
\text { - Male: } 257(66.93 \%) \\
\text { - Female: } 127 \\
(33.07 \%)\end{array}$ & $\begin{array}{l}\text { Design: Survey } \\
\text { Independent varia- } \\
\text { ble(s): } \\
\text { - Personal innovative- } \\
\text { ness } \\
\text { - Computer experience } \\
\text { Mediator variable(s): } \\
\text { - Computer self-efficacy } \\
\text { - Performance expecta- } \\
\text { tion }\end{array}$ & $\begin{array}{l}\text { - Personal innovativeness in the } \\
\text { domain of ICT was also identi- } \\
\text { fied as an important factor influ- } \\
\text { encing computer self-efficacy }(\beta \\
=.224, \mathrm{p}<.001) \text { for instructors. } \\
\text { - Computer experience was } \\
\text { significantly associated with } \\
\text { computer self-efficacy for in- } \\
\text { structors }(\beta=.223, \mathrm{p}<.001) \text { and } \\
\text { for learners }(\beta=.141, \mathrm{p}<.05) \text {. } \\
\text { - Computer self-efficacy partially }\end{array}$ \\
\hline
\end{tabular}




\begin{tabular}{|c|c|c|c|}
\hline $\begin{array}{l}\text { Authors } \\
\text { (Year) }\end{array}$ & $\begin{array}{l}\text { Sample Charac- } \\
\text { teristics }\end{array}$ & Study design & Finding \\
\hline & $\begin{array}{l}\text { Age: } \\
\text { Learner } \\
\text { - Lower-20: } 95 \\
(24.74 \%) \\
\text { - 20-25: } 262 \\
(68.23 \%) \\
\text { - 25-Upper: } 27 \\
\text { (7.03\%) } \\
\text { Mean: } 21.4 \\
\text { Instructors } \\
\text { - 20-29: } 171 \\
(48.44 \%) \\
\text { - 30-39: } 98(27.76 \%) \\
\text { - 40-49: } 54(15.30 \%) \\
\text { - 50-59: } 27(7.65 \%) \\
\text { - 50-Upper: } 3 \\
(0.85 \%) \\
\text { Mean: } 33.68\end{array}$ & $\begin{array}{l}\text { Dependent variable(s): } \\
\text { Behavioral intention to } \\
\text { use e-learning systems. }\end{array}$ & $\begin{array}{l}\text { mediated the effects of personal } \\
\text { experiences and innovativeness } \\
\text { in ICT on performance expecta- } \\
\text { tion in the case of instructors, } \\
\text { and it partially mediated their } \\
\text { effect on expectation in the case } \\
\text { of learners. }\end{array}$ \\
\hline
\end{tabular}

\subsection{Factors influencing self-efficacy in online learning}

The focus of the research question is on the factors that influence self-efficacy in the online learning environment. Self-efficacy perceptions can and do change as a result of environmental, cognitive, and behavioural effects that a person experiences in the course of everyday life [1], [3]. This study's findings define specific factors that literature reported as having a perceived effect on self-efficacy in the online learning environment. The result of Bates and Khasawneh [21] reported that previous online learning, instructor-acquired skill, instructor feedback, and online-learning system anxiety influenced students' self-efficacy in the context of online learning. These factors are consistent with the sources of self-efficacy introduced by Bandura [1] which states that self-efficacy expectations are based on four major sources of information: enactive mastery experience, vicarious experience, verbal persuasion as well as physiological and affective states. Findings are described on this topic as a set of categories which follow.

Online Learning Experience and Knowledge. Eight studies showed strong agreement on the effect of online learning experience and knowledge on self-efficacy. Choi, et al. [24] revealed that flow experience has a direct and indirect effect via attitude towards e-learning on technology self-efficacy in Enterprise Resource Planning training with a web-based e-learning (ERP) system usage. In a series of studies, Jashapara and Tai [10] , [54] demonstrated that computer experience influenced elearning system self-efficacy. Moreover, these findings suggested that personal innovativeness with information technology (IT) and computer playfulness also influenced e-learning system self-efficacy. Kim and Park [112] investigated factors influencing an individual's behavior to use e-learning through social-cognitive theory by examining the adoption of e-learning by instructors and learners. The results showed that computer experience significantly effected computer self-efficacy for learners. Me- 
anwhile, the personal innovativeness in the domain of information and communications technology (ICT) and computer experience was also identified as an important factor influencing computer self-efficacy for instructors. Prior, et al. [92] suggested that attitude and digital literacy has a significant positive effect on self-efficacy. Shen, et al. [61] explored the dimensions of online learning self-efficacy. The result demonstrated that online experience measured with the number of online courses was a significant predictor for self-efficacy to complete an online course. Song, Kalet and Plass [113] also examined the effects of medical clerkship students' prior knowledge, self-regulation, and motivation on learning performance in complex multimedia learning environments. The results showed that students with higher prior knowledge about a carotid disease case tended to report higher self-efficacy. Tang, Tang and Chiang [69] proposed an extended expectation-confirmation model (ECM) that explicitly incorporated experiential learning, perceived self-efficacy, and perceived usefulness to examine blog-continuance learning behavior intentions. The results demonstrated that blog learners' confirmation levels affected various learning beliefs, where the effect of perceived self-efficacy was the largest. Enactive mastery experiences are the most influential source of efficacy information because they provide the most authentic evidence of whether one can muster whatever it takes to succeed [1]. Contrarily, the experience of failure will result in recognition of self-efficacy, which leads to a lack of an attempt to complete tasks.

Feedback and Reward. Two studies reported the positive effect on self-efficacy when feedback and reward were presented. The finding from Liou, et al. [88] indicated that members of the Yamol online-test community improved knowledge sharing self-efficacy if they anticipated extrinsic rewards. Wang and $\mathrm{Wu}$ [30] suggested that students who received more elaborate feedback significantly increased their selfefficacy. The benefits of feedback and reward are the opportunity to discover whether they achieve their goals in learning.

Online Communication and Interactions. Six studies showed a strong agreement on the effect of online communication and interactions on self-efficacy. Cho and Cho [108] found that online-learner interaction with learner, content, and teacher are likely to demonstrate higher self-efficacy for learning and satisfaction with the course. Lim, et al. [80] also found the effect of learner-learner interaction on the computer and academic self-efficacy. Meanwhile, academic self-efficacy and computer self-efficacy were affected by content quality and system quality. Based on the community of inquiry framework, Lin et al. [76] investigated the relationship among forms of presence, self-efficacy, and training. The results showed that the teaching presence has a positive prediction on social presence, self-efficacy, and cognitive presence. Moreover, self-efficacy is a full mediator between social presence and cognitive presence. Tseng and Kuo [40] showed influences of community identity and interpersonal trust on knowledge-sharing behaviour through the mediation of social awareness and knowledge-sharing self-efficacy. Reychav, et al. [94] investigated the effect of social network on mobile collaboration with a focus on two aspects of social network mechanism, namely eigenvector centrality and network reciprocity. The results indicated that the network reciprocity formed through peer interactions between users in their daily lives can be leveraged when mobile devices are used in collaborative work. 
Shen [77] explored the impact of social interaction, perceptual learning, trust, a sense of community and self-efficacy for knowledge sharing among members in community. The empirical results showed that trust between members and perceptual learning has a significant effect to self-efficacy of knowledge sharing in virtual learning community. Vayre and Vonthron [103] reported that the sense of community plays an important role regarding self-efficacy in online education. Zang, et al. [56] reported effects of two environmental-communication factors, namely, psychological safetycommunication climate and perceived responsiveness on self-efficacy. The online communication and interaction not only allow learners to express themselves but also increase opportunities for learners to receive recognition of successful from each other. Online learning does not readily foster opportunities for observing peer success. Vicarious experience refers to one's observation of a role model performing a task successfully. Verbal persuasion can lead to higher self-efficacy by encouragements from others. Therefore, self-efficacy would be reduced if the learners fail to communicate and meet the performance of others. Verbal persuasion has limitations but can be powerful in conjunction with the role models of the individuals. One possibility for addressing the vicarious experience and verbal persuasion in online learning is for users to encourage communication and to share their successful experiences.

Social Influence. Three studies investigated the effect of social influence on selfefficacy. Social factor is defined as an individual's internalization of the reference group's subjective culture, and specific interpersonal agreements that the individual has made with others, in specific social situations [114]. Chiu and Tsai [66] revealed that the facilitating factor of social contexts in the workplace is an influential way of raising nurses' internet self-efficacy. In addition, the social factor plays an indirect role in the nurses' intentions to use web-based continuing learning through basic internet self-efficacy. Chu and Chu [35] proposed the role of collectivism and group potency at group level in predicting individual internet self-efficacy and individual elearning outcomes for people older than forty-five. The results showed that internet self-efficacy fully mediates the relationship between peer support and learners' persistence in e-learning. In addition, collectivism also moderates the relationship between peer support and internet self-efficacy. Chu [32] further indicated that family support had a most significant role in predicting the effects of e-learning, mediated by general and communication internet self-efficacy. In the gender model, men generally relied more on emotional support to enhance their communication-internet self-efficacy, whereas women showed more reliance on tangible support to increase their communication via the Internet. Social support is an important resource that can help individuals improve self-efficacy and handle stress. The last source of information is the direct effect physiological states can have on learners' self-efficacy. When people judge stress and anxiety, they depend on their state of physiological arousal. It is very likely that individuals will succeed if they are not in a state of aversive arousal [1]. In Chiu and Tsai [66] study, a head nurse or co-worker who is successful in utilizing online learning can serve as a role model for nursing staff.

Learner Motivation and Attitude. Three studies indicated that learner motivation and attitude was the main factor affecting the self-efficacy of the online learner. Motivation can be defined as the extent to which persistent effort is directed toward a 
goal [115]. Motivation can be determined intrinsically by individuals and externally by sources due to situational variables and environmental factors [116]. Hong, et al. [111] proposed that intrinsic motivation of Chinese learning could positively predict online learning self-efficacy. Law, et al. [39] reported that three motivating factors, namely, individual attitude and expectation, challenging goals, and social pressure and competition had a significant and positive relationship with self-efficacy. The $\mathrm{t}-$ test was used to compare the mean scores of constructs between male and female students. Male students are apparently more motivated by challenges, and they also showed a higher level of self-efficacy than female students. Wang, et al. [62] suggested that the level of motivation directly influenced the level of technology selfefficacy. Self-efficacy and motivation have a complex interrelationship. It is likely that each influences or supports the other. However, motivation may be strong enough to overcome a weaker sense of self-efficacy.

\subsection{Limitation}

The main limitations are the fact that only published papers written in English between 2005 and 2017 were included in the review process. Most of the selected studies applied survey design. More rigorous research design, such as incorporating a comparison group, is needed to conclude that the reported literature conclusively had an effect on self-efficacy in online learning.

\section{Conclusion}

Self-efficacy is the key to success in all activities including online learning. Hence, the understanding of the source of self-efficacy in online learning context is important. As found in this systematic review, many researchers focused on the investigation of various factors that influenced learner self-efficacy in online learning context. These various factors were source of self-efficacy in online learning context as follows: online learning experience and knowledge, feedback and reward, online communication and interactions, social influence, and learner motivation and attitude. Moreover, the results of this review can be guidance in further research for design online learning to enhance self-efficacy of learner.

\section{$5 \quad$ References}

[1] Bandura, A. (1997). Self-efficacy: The exercise of control. New York: W.H. Freeman.

[2] Bandura, A. (1988). Perceived self-efficacy: Exercise of control through self-belief. In J. Dauwalder, P. Perrez, M. and Hobi, V. (Eds.), Annual series of European research in behavior therapy, 2: 27-59

[3] Schunk, D. (1991). Self-efficacy and academic motivation. Educational Psychologist, 26 (3-4): 207-231 https://doi.org/10.1080/00461520.1991.9653133 
[4] Hodges, C. B. (2008). Self-efficacy in the context of online learning environments: A review of the literature and directions for research. Performance Improvement Quarterly, 20(3-4): 7-25 https://doi.org/10.1002/piq.20001

[5] Cho, M. H. and Jonassen, D. (2009). Development of the Human Interaction Dimension of the Self-Regulated Learning Questionnaire in Asynchronous Online Learning Environments. Educational Psychology, 29(1): 117-138 https://doi.org/10.1080/014434108 02516934

[6] Cho, M. H. Demei, S. and Laffey, J. (2010). Relationships between Self-Regulation and Social Experiences in Asynchronous Online Learning Environments. Journal of Interactive Learning Research, 21(3): 297-316

[7] Ali, R. and Leeds, E. M. (2009). The Impact of Face-to-Face Orientation on Online Retention: A Pilot Study. Online Journal of Distance Learning Administration, 12(4):

[8] Lee, Y. and Choi, J. (2011). A review of online course dropout research: Implications for practice and future research. Educational Technology Research and Development, 59: 593-618 https://doi.org/10.1007/s11423-010-9177-y

[9] Moher, D. Liberati, A. Tetzlaff, J. and Altman, D. G. (2009). Preferred reporting items for systematic reviews and meta-analyses: the PRISMA statement, Ann. Intern. Med., 151(4): 264-269 https://doi.org/10.7326/0003-4819-151-4-200908180-00135

[10] Jashapara, A. and Tai, W. C. (2006). Understanding the complexity of human characteristics on e-learning system: an integrated study of dynamic individual differences on user perceptions of ease of use. Knowledge Management Research \& Practice, 4: 227-239 https://doi.org/10.1057/palgrave.kmrp.8500099

[11] Agarwal, R. and Prasad, J. (1998). A conceptual and operational definition of personal innovativeness in the domain of information technology. Information Systems Research, 9(2): 204-215 https://doi.org/10.1287/isre.9.2.204

[12] Webster, J. and Martocchio, J. J. (1992). Microcomputer playfulness - development of a measure with workplace implications. MIS Quarterly, 16(2): 201-226 https://doi.org/10.2307/249576

[13] Potosky, D. and Bobko, P. (1998). The computer understanding and experience scale: a self-report measure of computer experience. Computers in Human Behavior, 14(2): 337348 https://doi.org/10.1016/S0747-5632(98)00011-9

[14] Bozionelos, N. (2004). Socio-economic background and computer use: the role of computer anxiety and computer experience in their relationship. International Journal of HumanComputer Studies, 61(5): 725-746 https://doi.org/10.1016/j.ijhcs.2004.07.001

[15] Hasan B. and Ali J. M. H. (2004). An empirical examination of a model of computer learning performance. Journal of Computer Information Systems, 44(4): 27-33

[16] Marakas, G. M. Yi, M. Y. and Johnson, R. D. (1998). The multilevel and multifaceted character of computer self- efficacy: toward clarification of the construct and an integrative framework for research. Information Systems Research, 9(2): 126-163 https://doi.org/10.1287/isre.9.2.126

[17] Thatcher, J. B. and Perrewe, P. L. (2002). An empirical examination of individual traits as antecedents to computer anxiety and computer self-efficacy. MIS Quarterly, 26(4): 381396 https://doi.org/10.2307/4132314

[18] Barbeite, F. G. and Weiss, E. M. (2004). Computer self-efficacy and anxiety scales for an Internet sample: testing measurement equivalence of existing measures and development of new scales. Computers in Human Behavior, 20(1): 1-15 https://doi.org/10.1016/S0747$\underline{5632(03) 00049-9}$ 
[19] Venkatesh, V. and Davis, F. D. (1996). A model of the antecedents of perceived ease of use: development and test. Decision Sciences, 27(3): 451-481 https://doi.org/10.1111/ j.1540-5915.1996.tb01822.x

[20] Venkatesh, V. and Davis, F. D. (2000). A theoretical extension of the Technology Acceptance Model: four longitudinal field studies. Management Science, 46(2): 186-204 https://doi.org/10.1287/mnsc.46.2.186.11926

[21] Bates, R. and Khasawneh, S. (2007). Self-efficacy and college student' perceptions and use of online learning systems. Computers in Human Behavior, 23: 175-191 https://doi.org/10.1016/j.chb.2004.04.004

[22] Compeau, D. and Higgins, C. A. (1995). Computer self-efficacy: Development of a measure and initial test. MIS Quarterly, 19(2): 189-211 https://doi.org/10.2307/249688

[23] Compeau, D. Higgins, C. A. and Huff, S. (1999). Social cognitive theory and individual reactions to computing technology: A longitudinal study. MIS Quarterly, 23(2): 145-158 https://doi.org/10.2307/249749

[24] Choi, D. H. Kim, J. and Kim, S. H. (2007). ERP training with a web-based electronic learning system: The flow theory perspective. Int. J. Human-Computer Studies, 65: 223243 https://doi.org/10.1016/j.ijhcs.2006.10.002

[25] Volery, T. and Lord, D. (2000). Critical success factors in online education. The International Journal of Educational Management, 14(5): 216-223 https://doi.org/10.1108/095 13540010344731

[26] Wang, Y.S. (2003). Assessment of learner satisfaction with asynchronous electronic learning systems. Information \& Management, 41: 75-86 https://doi.org/10.1016/S03787206(03)00028-4

[27] Taylor, S. and Todd, P. A. (1995). Understanding information technology usage: a test of competing models. Information System Research, 6(2): 144-176 https://doi.org/10.1287/ isre.6.2.144

[28] Novak, T. Hoffman, D. and Yung, Y. (2000). Measuring the customer experience in online environments: a structural modeling approach. Marketing Science, 19(1): 22-42 https://doi.org/10.1287/mksc.19.1.22.15184

[29] Hollenbeck, J. R. and Brief, A. P. (1987). The effects of individual differences and goal origin on goal setting and performance. Organizational Behavior and Human Decision Processes, 40: 392-414 https://doi.org/10.1016/0749-5978(87)90023-9

[30] Wang, S. L. and Wu, P. Y. (2008). The role of feedback and self-efficacy on web-based learning: The social cognitive perspective. Computers \& Education, 51: 1589-1598 https://doi.org/10.1016/j.compedu.2008.03.004

[31] Wang, S. L. and Lin, S. S. J. (2000). The cross-cultural validation of motivated strategies for learning questionnaire. Paper presented at the 2000 Annual Conference of American Psychological Association, Washington DC.

[32] Chu, R. J. (2010). How family support and Internet self-efficacy influence the effects of elearning among higher aged adults-Analyses of gender and age differences. Computers \& Education, 55: 255-264 https://doi.org/10.1016/j.compedu.2010.01.011

[33] Tsai, M.J. and Tsai, C.C. (2003). Information searching strategies in web-based science learning: The role of internet self-efficacy. Innovations in Education and Teaching International, 40: 43-50 https://doi.org/10.1080/1355800032000038822

[34] Shin, N. and Chan, J. K. Y. (2004). Direct and indirect effects of online learning on distance education. British Journal of Educational Technology, 25(3): 275-288 https://doi.org/10.1111/j.0007-1013.2004.00389.x 
[35] Chu, R. J. and Chu, A. Z. (2010). Multi-level analysis of peer support, Internet selfefficacy and e-learning outcomes-The contextual effects of collectivism and group potency. Computers \& Education. 55: 145-154 https://doi.org/10.1016/j.compedu.2009.12.011

[36] Schepers, J. Jong, A. Wetzels, M. and Ruyter, K. (2008). Psychological safety and social support in groupware adoption: A multi-level assessment in education. Computers \& Education, 51(2): 757-775 https://doi.org/10.1016/j.compedu.2007.08.001

[37] Schaubroeck, J. Lam, S. S. K. and Cha, S. E. (2007). Embracing transformational leadership: Team value and the impact of leader behavior on team performance. Journal of Applied Psychology, 92(4): 1020-1030 https://doi.org/10.1037/0021-9010.92.4.1020

[38] Lent, R. W. Schmidt, J. and Schmidt, L. (2006). Collective efficacy beliefs in student work relation to self-efficacy, cohesion and performance. Journal of Vocational Behavior, 68: 73-84 https://doi.org/10.1016/j.jvb.2005.04.001

[39] Law, K. M. Y. Lee, V. C. S. and Yu, Y. T. (2010). Learning motivation in e-learning facilitated computer programming courses. Computers \& Education, 55: 218-228 https://doi.org/10.1016/j.compedu.2010.01.007

[40] Tseng, F. C. and Kuo, F. Y. (2010). The way we share and learn: An exploratory study of the self-regulatory mechanisms in the professional online learning community. Computer in human behavior, 26: 1043-1053 https://doi.org/10.1016/j.chb.2010.03.005

[41] Triandis, H. C. (1995). Individualism and collectivism., Boulder, Colo: Westview.

[42] Leana, C. R. and van Buren, H. J. III. (1999). Organizational social capital and employment practices. Academy of Management Review, 24(3): 538-555 https://doi.org/10.5465/ amr.1999.2202136

[43] Jarvenpaa, S. L. Knoll, K. and Leidner, D. E. (1998). Is anybody out there? Antecedents of trust in global virtual teams. Journal of Management Information Systems, 14(4): 29-64 https://doi.org/10.1080/07421222.1998.11518185

[44] Ridings, C. M. Gefen, D. and Arinze, B. (2002). Some antecedents and effects of trust in virtual communities. Journal of Strategic Information Systems, 11: 271-295 https://doi.org/10.1016/S0963-8687(02)00021-5

[45] Leimeister, J. M. Ebner, W. and Krcmar, H. (2005). Design, implementation, and evaluation of trust-supporting components in virtual communities for patients. Journal of Management Information Systems, 21(4): 101-135 https://doi.org/10.1080/07421222.2 005.11045825

[46] Daniel, B. Schwier, R. A. and McCalla, G. (2003). Social capital in virtual learning Communities and Distributed Communities of Practice. Canadian Journal of Learning and Technology, 29(3): 113-139

[47] Cross, R. and Borgatti, S. P. (2004). The ties that share: Relational characteristics that facilitate information seeking. In Huysman, M. and Wulf V. (Eds.), Social capital and information technology, Cambridge, Mass: The MIT Press.

[48] Cabrera, A. and Cabrera, E. F. (2002). Knowledge-sharing dilemmas. Organization Studies, 23(5): 687-710 https://doi.org/10.1177/0170840602235001

[49] Lu, L. Leung, K. and Koch, P. T. (2006). Managerial knowledge sharing: The role of individual, interpersonal, and organizational factors. Management and Organization Review, 2(1): 15-41 https://doi.org/10.1111/j.1740-8784.2006.00029.x

[50] Kuo, F. Y. and Young, M. L. (2008a). Predicting knowledge sharing practices through intention: A test of competing models. Computers in Human Behavior, 24(6): 2697-2722 https://doi.org/10.1016/j.chb.2008.03.015

[51] Kuo, F. Y. and Young, M. L. (2008b). A study of the intention-action gap in knowledge sharing practices. Journal of the American Society for Information Science and Technology, 59(8): 1224-1237 https://doi.org/10.1002/asi.20816 
[52] Carroll, J. M. Choo, C. W. Dunlap, D. R. Isenhour, P. L. Kerr, S. T. and MacLean, A. (2003). Knowledge management support for teachers. Educational Technology, Research and Development, 51(4): 42-64 https://doi.org/10.1007/BF02504543

[53] Imants, J. (2003). Two basic mechanisms for organizational learning in schools. European Journal of Teacher Education, 26(3): 293-311 https://doi.org/10.1080/0261976032000 $128157 \mathrm{~A}$

[54] Jashapara, A. and Tai, W. C. (2011). Knowledge mobilization through e-learning system: Understanding the mediating roles of Self-Efficacy and Anxiety on perceptions of ease of use. Information Systems Management, 28(1): 71-83 https://doi.org/10.1080/105805 30.2011 .536115

[55] Thatcher, J. B. and Perrewe, P. L. (2002). An empirical examination of individual traits as antecedents to computer anxiety and computer self-efficacy. MIS Quarterly, 26(4): 381396 https://doi.org/10.2307/4132314

[56] Zhang, Y. Fang, Y. Wei, K. K. and Wang, Z. (2012). Promoting the intention of students to continue their participation in e-learning systems. Information Technology \& People, 25(4): 356-375 https://doi.org/10.1108/09593841211278776

[57] Gibson, C. B. and Gibbs, J. L. (2006). Unpacking the concept of virtuality: the effects of geographic dispersion, electronic dependence, dynamic structure, and national diversity on team innovation. Administrative Science Quarterly, 51(3): 451-95 https://doi.org/10.2189/ asqu.51.3.451

[58] Kankanhalli, A. Tan, B. C. Y. and Wei, K. K. (2005). Contributing knowledge to electronic knowledge repositories: an empirical investigation, MIS Quarterly, 29(1): 113-143 https://doi.org/10.2307/25148670

[59] Bhattacherjee, A. (2001a). An empirical analysis of the antecedents of electronic commerce service continuance., Decision Support Systems, 32: 201-214 https://doi.org/10.1016/S0167-9236(01)00111-7

[60] Chen, I.Y. (2007). The factors influencing members continuance intentions in professional virtual communities - a longitudinal study. Journal of Information Science, 33(4): 451-467 https://doi.org/10.1177/0165551506075323

[61] Shen, D. Cho, M. H. Tsai, C. L. and Marra, R. (2013). Unpacking online learning experiences: Online learning self-efficacy and learning satisfaction., The Internet and Higher Education, 19: 10-17 https://doi.org/10.1016/j.iheduc.2013.04.001

[62] Wang, C. H. Shannon, D. M. and Ross, M. E. (2013). Student' characteristics, selfregulated learning, technology self-efficacy, and course outcomes in online learning. Distance Education, 34(3): 302-323 https://doi.org/10.1080/01587919.2013.835779

[63] Artino, and McCoach. (2008). Development and initial validation of the online learning value and self-efficacy scale. Journal of Educational Computing Research, 38, 279-303. https://doi.org/10.2190/EC.38.3.c

[64] Miltiadou, M. and Yu, C. H. (2000). Validation of the online technologies self-efficacy scale (OTSES). Pheonix AZ: Arizona state University

[65] Frey, A. Yankelov, P. and Faul, A. C. (2003). Student perceptions of web-assisted teaching strategies., Journal of Social Work Education, 39: 443-457 https://doi.org/10.1080/ 10437797.2003.10779148

[66] Chiu, Y. L. and Tsai, C. C. (2014). The roles of social factor and internet self-efficacy in nurses' web-based continuing learning. Nurse Education Today, 34: 446-450 https://doi.org/10.1016/j.nedt.2013.04.013

[67] Thompson, R.L. Higgins, C.A. and Howell, J.M. 1991. Personal computing: toward a conceptual model of utilization. MIS Quarterly 15(1): 125-143 https://doi.org/10.2307/249443 
[68] Liang, J.C. Wu, S.H. and Tsai, C.C. 2011. Nurses' Internet self-efficacy and attitudes toward web-based continuing learning. Nurse Education Today 31(8): 768-773 https://doi.org/10.1016/j.nedt.2010.11.021

[69] Tang, J.T.E. Tang, T.I. and Chiang, C.H. (2014). Blog learning: effects of users' usefulness and efficiency towards continuance intention. Behavior \& Information Technology 33(1): 36-50 https://doi.org/10.1080/0144929X.2012.687772

[70] Bhattacherjee, A. (2001b). Understanding information systems continuance: an expectation-confirmation model. MIS Quarterly, 25: 351-370 https://doi.org/10.2307/3250921

[71] Spreng, R. A. and Olshavsky, R. W. (1993). A desires congruency model of consumer satisfaction. Journal of the Academy of Marketing Science, 21: 169-177 https://doi.org/10.1177/0092070393213001

[72] Kolb, D. A. (1984). Experiential learning: experience as the source of learning and development. Englewood Cliffs, NJ: Prentice Hall.

[73] Duncan, T. G. and McKeachie, W. J. (2005). The making of the motivated strategies for learning questionnaire. Educational Psychologist, 40: 117-128 https://doi.org/10.1207/ s15326985ep4002 6

[74] Davis, F. Bagozzi, R. and Warshaw, P. (1989). User acceptance of computer technology: a comparison of two theoretical models. Management Science, 35(8): 982-1003 https://doi.org/10.1287/mnsc.35.8.982

[75] Lee, J. K. and Hwang, C. Y. (2007). The effects of computer self-efficacy and learning management system quality on e-Learner's satisfaction. In: Cameron, L. Voerman, A. and Dalziel, J. eds. Proceedings of the 2007 European LAMS Conference: designing the future of learning, Greenwich: LAMS Foundation, 73-79

[76] Lin, S. Hung, T. C. and Lee, C. T. (2015). Revalidate forms of presence in training effectiveness: Mediating effect of Self-Efficacy. Journal of Educational Computing Research, 53(1): 32-54 https://doi.org/10.1177/0735633115588772

[77] Shen, B. (2015). An Empirical Study on Influencing Factors of Knowledge Sharing in Virtual Learning Community. The Open Cybernetics \& Systemics Journal, 9: 2332-2338 https://doi.org/10.2174/1874110X01509012332

[78] Koh, J. and Kim, Y. G. (2003). Sense of virtual community: A conceptual framework and empirical validation. International Journal of Electronic Commerce, 8: 75-94 https://doi.org/10.1080/10864415.2003.11044295

[79] Hsu, M. H. Ju, T. L. Yen, C. H. and Chang, C. M. (2007). Knowledge sharing behavior in virtual communities: The relationship between trust, self-efficacy, and outcome expectations. International Journal of Human-Computer Studies, 65: 153-169 https://doi.org/10.1016/j.ijhcs.2006.09.003

[80] Lim, K. Kang, M. and Park, S. Y. (2016). Structural relationships of environments, Individuals, and learning outcomes in Korean online university settings, International Review of Research in Open and Distributed Learning, 17(4): 315-330 https://doi.org/10.19173/ irrodl.v17i4.2500

[81] An, B. K. (2008). Development of evaluation criteria for interactions in e-learning for public schools. Keimyung University, Daegu, Korea.

[82] Kettinger, J. W. and Lee, L. L. (1997). Pragmatic perspectives on the measurement of information systems service quality. MIS Quarterly, 21(2): 223-240 https://doi.org/10.2307/ 249421

[83] Lee, Y. W. Strong, D. M. Kahn, B. K. and Wang, R. Y. (2002). AIMQ: a methodology for information quality assessment., Information \& Management, 40: 133-146 https://doi.org/10.1016/S0378-7206(02)00043-5 
[84] Gu, J. C. Lee, S. C. Kim, N. H. and Suh, Y. H. (2006). Factors affecting user acceptance in mobile banking: An empirical study using extended tam and trust. Journal of Management \& Information, 16(2): 159-181

[85] Stein, J. J. (1997). Asynchronous computer conferencing as a supplement to classroom instruction in higher education: The impact of selected learner characteristics on user satisfaction and the amount of interaction. Wayne State University, Michigan, the United States.

[86] Lee, Y. C. (2006). An empirical investigation into factors influencing the adoption of an elearning system. Online Information Review, 30(5): 517-541 https://doi.org/10.1108/1468 4520610706406

[87] Flowers, L. O. (2011). Exploring HBCU student academic self-efficacy in online STEM courses. The Journal of Human Resource and Adult Learning, 7(2): 139-145

[88] Liou, D. K. Chih, W. H. Yuan, C. Y. and Lin, C. Y. (2016). The study of the Antecedents of Knowledge sharing behavior: The empirical study of Yamol online test community, Internet Research, 26(4): 845-868

[89] Bock, G. W. Zmud, R. W. Kim, Y. G. and Lee, J. N. (2005). Behavioral intention formation in knowledge sharing: examining the roles of extrinsic motivators, socialpsychological forces, and organizational climate. MIS Quarterly, 29(1): 87-111 https://doi.org/10.2307/25148669

[90] Lin, M.J.J. Hung, S.W. and Chen, C.J. (2009). Fostering the determinants of knowledge sharing in professional virtual communities. Computers in Human Behavior, 25(4): 929-939 https://doi.org/10.1016/j.chb.2009.03.008

[91] Koh, J. and Kim, Y. G. (2004). Knowledge sharing in virtual communities: an Ebusiness perspective. Expert Systems with Applications, 26(2): 155-166 https://doi.org/10.1016/S09 57-4174(03)00116-7

[92] Prior, D. D. Mazanov, J. Meacheam, D. Heaslip, H. and Hanson, J. (2016). Attitude, digital literacy and self-efficacy: Flow-on effects for online learning behavior. The Internet and Higher Education, 29: 91-97 https://doi.org/10.1016/j.iheduc.2016.01.001

[93] Ng, W. (2012). Can we teach digital natives digital literacy? Communication Education, 59(3): 1065-1078

[94] Reychav, I. Ndicu, M. and Wu, D. (2016). Leveraging social networks in the adoption of mobile technologies for collaboration. Computer Human Behavior, 58: 443-453 https://doi.org/10.1016/j.chb.2016.01.011

[95] Johnson, R. D. and Marakas, G. M. (2000). Research report: the role of behavioral modeling in computer skills acquisition: toward refinement of the model. Information Systems Research, 11(4): 402-417 https://doi.org/10.1287/isre.11.4.402.11869

[96] Davis, F. D. Bagozzi, R. P. and Warshaw, P. R. (1992a). Extrinsic and intrinsic motivation to use computers in the workplace1. Journal of Applied Social Psychology, 22(14): 1111$1132 \mathrm{https} / / /$ doi.org/10.1111/j.1559-1816.1992.tb00945.x

[97] Song, H. S. Kalet, A. L. and Plass, J. L. (2011). Assessing medical students' selfregulation as aptitude in computer based learning., Advances in Health Sciences Education, 16: 97-107 https://doi.org/10.1007/s10459-010-9248-1

[98] Vandewalle, D. (1997). Development and validation of a work domain goal orientation instrument. Educational and Psychological Measurement, 57(6): 995-1015 https://doi.org/10.1177/0013164497057006009

[99] Bong, M. (2001). Between- and within-domain relations of academic motivation among middle and high school students: Self-efficacy, task value, and achievement goals. Journal of Educational Psychology, 93(1): 23-34 https://doi.org/10.1037/0022-0663.93.1.23 
[100] Bong, M. (2004). Academic motivation in self-efficacy, task value, achievement goal orientations, and attributional beliefs. Journal of Educational Research, 97(6): 287-297 https://doi.org/10.3200/JOER.97.6.287-298

[101] Pintrich, P. R. and De Groot, E. V. (1990). Motivational and self-regulated learning components of classroom academic performance. Journal of Educational Psychology, 82(1): 33-40 https://doi.org/10.1037/0022-0663.82.1.33

[102] Charlin, B. Brailovsky, C. Roy, L. Goulet, F. and van der Vleuten, C. (2000). The script concordance test: A tool to assess the reflective clinician. Teaching and Learning in Medicine, 12(4): 189-195 https://doi.org/10.1207/S15328015TLM1204 5

[103] Vayre, E. and Vonthron, A. M. (2016). Psychological engagement of students in distance and online learning: Effects of self-efficacy and psychosocial processes. Journal of Educational Computing Research, 55(2): 197 - 218 https://doi.org/10.1177/0735633116656849

[104] Rovai, A. P. (2002a). Development of an instrument to measure classroom community. The Internet and Higher Education, 5(3): 197-211 https://doi.org/10.1016/S10967516(02)00102-1

[105] Rovai, A. P. Wighting, M. J. and Lucking, R. (2004). The classroom and school community inventory: Development, refinement and validation of a self-report measure for educational research. The Internet and Higher Education, 7(4): 263-280 https://doi.org/10.1016/j.iheduc.2004.09.001

[106] Vonthron, A. M. Lagabrielle, C. and Pouchard, D. (2007). Professional training maintenance: Effects of motivational, cognitive and social factors. L'orientation Scolaire et Professionnelle, 36(3): 401-420 https://doi.org/10.4000/osp.1481

[107] Brault-Labbe, A. and Dube, L. (2008). Academic engagement, over-engagement and under-engagement at college: Toward a better understanding of students' wellbeing. Revue des sciences de l'education, 34(3): 729-751

[108] Cho, M. H. and Cho, Y. J. (2017). Self-regulation in three types of online interaction: a scale development. Distance Education, 38(1): 70-83 https://doi.org/10.1080/01587919. $\underline{2017.1299563}$

[109] Pintrich, P. R. Smith, D. A. F. Garcia, T. and Mckeachie, W. J. (1993). Reliability and predictive validity of the motivated strategies for learning questions (MSLQ). Educational and Psychological Measurement, 53: 801-813 https://doi.org/10.1177/00131644930530 03024

[110] Lin, Y.M. Lin, G.Y. and Laffey, J.M. (2008). Building a social and motivational framework for understanding satisfaction in online learning. Journal of Educational Computing Research, 38(1): 1-27 https://doi.org/10.2190/EC.38.1.a

[111] Hong, J. C. Hwang, M. Y. Tai, K. H. and Lin, P. H. (2017). Intrinsic motivation of Chinese learning in predicting online learning self-efficacy and flow experience relevant to students' learning progress. Computer assisted language learning. 30(6): 552-574 https://doi.org/10.1080/09588221.2017.1329215

[112] Kim, B. and Park, M. J. (2017). Effect of personal factors to use ICTs on e-learning adoption: comparison between learner and instructor in developing countries. Information Technology for Development, 1-27

[113] Song, H. S. Kalet, A. L. and Plass, J. L. Interplay of prior knowledge, self-regulation and motivation in complex multimedia learning environments. Journal of Computer Assisted Learning, 32: 31-50 https://doi.org/10.1111/jcal.12117

[114] Triandis, H.C. (1980). Values, attitudes, and interpersonal behavior, in: M.M. Page Ed., Nebraska Symposium on Motivation, 1979: Beliefs, Attitudes, and Values, Univ. Nebraska Press, Lincoln, 195-259 
[115] Johns, G. (1996). Organizational behaviour: Understanding and managing life at work, 4th ed., New York: Harper Collins.

[116] Ryan, R. M. and Deci, E. L. (2000). Self-determination theory and the facilitation of intrinsic motivation, social development and well-being, American Psychologist, 55: 68-78 https://doi.org/10.1037/0003-066X.55.1.68

\section{Authors}

Chattavut Peechapol is currently a Ph.D. Student in Technopreneurship and Innovation Management Program at the Chulalongkorn University, Bangkok 10330, Thailand. He also works at the Southeast Asia University, Bangkok, Thailand. His main research interests in educational innovation and information technology management. E-mail: provut@gmail.com

Jaitip Na-Songkhla is currently an Associate Professor in the Department of Educational Technology and Communications, Chulalongkorn University, Bangkok 10330, Thailand. Her main research interests are educational technology development and educational policy studies. E-mail: jaitipn@gmail.com

Siridej Sujiva is currently an Associate Professor in the Department of Educational Research and Psychology, Faculty of Education, Chulalongkorn University, Bangkok 10330, Thailand. His main research interests are educational measurement and evaluation. E-mail: ssiridej@chula.ac.th

Arthorn Luangsodsai is currently a Lecturer in the Department of Mathematics and Computer Science, Faculty of Science, Chulalongkorn University, Bangkok 10330, Thailand. His main research interests model-based slicing, program slicing, software engineering, outlier detection, information management, databases and cloud computing. E-mail: arthorn.1@chula.ac.th

Article submitted 04 February 2018. Resubmitted 02 March 2018. Finala cceptance 23 March 2018. Final version published as submitted by the authors. 